\title{
Vacunación en el personal sanitario como medida preventiva frente al riesgo biológico y la desinformación
}

\section{Vaccination of health professionals as a preventive measure against biological risk and disinformation}

Juan José Tejada-Péreza , Juan José Vázquez-Vicentea, Francisco Gabriel Martín-Martína, María Renée Herrera-Burgosa ${ }^{a}$ Joaquín Jesús Molina-Díaza , María Ángeles Lucerna-Méndeza

a Servicio de Prevención, Agencia Pública Sanitaria Poniente, España

\section{Resumen}

Introducción: La vacunación es una herramienta esencial en la prevención de enfermedades altamente contagiosas. Actualmente, dicho procedimiento comienza a ser menos empleado debido, entre otros, a diversos factores económicos y sociales, que han promovido la reaparición de patologías que estaban prácticamente desaparecidas. Objetivos: Definir el estado inmunitario de los trabajadores sanitarios del Hospital de Poniente de la provincia de Almería y observar la eficacia de las vacunas administradas por el Servicio de Prevención en el personal sanitario no inmunizado. Metodología: Estudio descriptivo en el que se evalúa mediante serología, la inmunidad frente al sarampión, la rubéola, la parotiditis, la varicela y la hepatitis B previa y posterior a la administración de vacunas en el personal sanitario $(n=923)$. Resultados: Se observa un alto porcentaje de la población trabajadora sanitaria con anticuerpos protectores frente a los microorganismos del estudio, ya sea por una correcta pauta vacunal en la infancia como por la posterior vacunación por parte del Servicio de Prevención (alta tasa de seroconversión). Conclusión: El estudio señala la importancia de la vacunación como medida preventiva contra las enfermedades estudiadas, por lo que justifica la necesidad de poner en marcha campañas informativas que refuercen su uso.

Palabras clave: vacunación; personal de salud; serología; salud laboral.

\begin{abstract}
Introduction: Vaccination is an essential tool in the prevention of highly contagious diseases. Currently, this procedure is becoming less widely used, due to various economic and social factors, among others, which have encouraged the reappearance of pathologies that were virtually disappeared. Objectives: To define the immune status of the health workers of the Hospital de Poniente and monitoring the effectiveness of vaccines administered by the Prevention Service on non-immunized health personnel. Methodology: A descriptive study evaluating by serology the immunity against measles, rubella, mumps, varicella and hepatitis $B$ prior to and after the administration of vaccines to health personnel $(n=923)$. Results: A high percentage of the health worker population with protective antibodies against the microorganisms in the study was observed, either due to a correct vaccination schedule in childhood or due to subsequent vaccination by the Prevention Service (high rate of seroconversion). Conclusion: The study re-emphasizes the importance of vaccination as a preventive measure against the diseases analysed, justifying the need to launch information campaigns to strengthen their use.
\end{abstract}

Key Words: vaccination; health personnel; serology; occupational health. 


\section{Introducción}

$\mathrm{D}$ esde su aparición, las vacunas se presentan como la principal medida de prevención frente a diversos patógenos. Éstas actúan mediante la exposición del individuo a cepas del microorganismo con menor virulencia que la original, dando lugar a las vacunas atenuadas, y a antígenos aislados de los patógenos sin capacidad infecciosa por sí mismos, produciendo las conocidas como vacunas inactivadas (Arrazola Martínez, de Juanes Pardo, \& García de Codes llario, 2015).

Gracias al proceso de vacunación se ha producido una inmunidad generalizada en la población, cuyo resultado ha supuesto que enfermedades que antes eran muy comunes hayan dejado de presentarse. Esto ha justificado que durante el último siglo la vacunación se haya extendido a la población general, siendo universalizada y gratuita en España, generándose múltiples vacunas dirigidas a los microorganismos más prevalentes de nuestro medio.

Por su gran difusión histórica, la vacunación ha generado inmunidad tanto de forma directa como indirecta, mediante lo que se conoce como inmunidad de grupo (o inmunidad de rebaño). A través de este tipo de inmunización, un individuo que tras la vacunación no desarrolla memoria inmunitaria o que no se ha vacunado, puede evitar infectarse con el patógeno al estar protegido el resto de la población. Este fenómeno, por tanto, dificulta la transmisión de enfermedades que utilizan a otros individuos como vectores (Amela, 2004).

En la actualidad, diferentes tipos de vacunas se programan para la población infantil, dando lugar al calendario vacunal que es ofertado a nivel nacional (Ministerio de Sanidad, Consumo y Bienestar Social, 2019). Durante la infancia, no toda la población se vacuna, siendo necesario en muchos casos iniciar la vacunación durante la vida adulta (Ministerio de Sanidad, Consumo y Bienestar Social, 2018). Además, la respuesta inmunitaria puede disminuir con el tiempo, a pesar de estar vacunados, por lo que se considera a los trabajadores sanitarios un grupo laboral cuya exposición frente a agentes infecciosos hace necesario dotarles de una cobertura vacunal lo más completa posible. Por ello, en el caso de este colectivo profesional y aún teniendo en cuenta que la vacunación no tiene un carácter obligatorio, sí es altamente recomendable su vacunación con el objeto de protegerlos frente a patógenos como sarampión, rubéola, parotiditis, varicela o hepatitis $B$ (Ministerio de Sanidad, Servicios Sociales e Igualdad, 2017).
A pesar de la demostrada eficacia de la vacunación, una nueva corriente ideológica conocida como el "Movimiento anti-vacunas" promueve el abandono de su aplicación de manera sistemática (Rosell Aguilar, 2017; Salleras, 2018). La principal causa que impulsa a este grupo ha sido la gran desinformación de la población general, apoyando el rechazo a la vacunación por temor a la gravedad de algunos efectos adversos raros que pueden presentarse tras su administración (cuadros pseudo-infecciosos o reacciones alérgicas, entre otros). Así mismo, esta corriente ideológica se ve reforzada por determinadas especulaciones con gran difusión a través de diferentes medios de comunicación, tales como el hecho de relacionar el autismo con la vacunación, dando así mayor relevancia al riesgo mínimo al que se exponen los vacunados, en comparación con el mayor peligro que conlleva el desarrollo de algunas de estas enfermedades (Torres González, 2018).

En los últimos años se está comprobando que el descenso de vacunación en la población infantil coincide con el aumento de actividad del "Movimiento antivacunas", produciéndose un gran impacto en la salud, así como en el aspecto económico y social de una generación que ya llega a la madurez (Kew, 2010; Shepard, 2006; Zanetti, Van Damme, \& Shouval, 2008). Este factor, unido al uso de vacunas con lotes defectuosos en ciertos años, así como, la respuesta inmunitaria dependiente de sexo o edad del individuo, entre otros, ha ocasionado la aparición de subgrupos poblacionales que no presentan protección frente a patógenos, como el sarampión, la rubéola o la parotiditis, que anteriormente se encontraban prácticamente desaparecidos, poniendo en riesgo la pérdida de inmunidad colectiva referida anteriormente.

Debido a que en nuestro centro de trabajo, los profesionales sanitarios atienden a una gran diversidad de población con orígenes étnicos y estados serológicos muy diversos, desde la consulta de Salud Laboral se intenta realizar la mayor cobertura vacunal posible al personal sanitario. A su vez, transmitimos las ventajas que conlleva una correcta vacunación ante un posible contacto de riesgo, frente a la desinformación generalizada que puedan recibir de la comunidad.

Elaboramos este estudio para conocer la protección inmunitaria que presenta nuestro personal sanitario, la cual puede haber descendido debido a los motivos anteriormente expuestos, así como a otros motivos de recienteaparición, tales como los recortes presupuestarios en el ámbito sanitario sufridos en España en los últimos años 0 en algunos casos al desabastecimiento de lotes concretos de vacunas. 


\section{Objetivos}

1. Describir el estado serológico de la plantilla sanitaria del centro Hospital de Poniente de la provincia de Almería.

2. Establecer una comparativa generacional entre los trabajadores y su estado inmunitario.

3. Fijar si las pautas vacunales aplicadas en los últimos años producen una seroconversión en la población trabajadora.

4. Concluir si son necesarias nuevas estrategias que aumenten la vacunación del personal sanitario.

\section{Material y métodos}

\section{Diseño}

Se trata de un estudio descriptivo desarrollado durante el año 2019 en el Hospital de Poniente, ubicado en la provincia de Almería, Andalucía (España).

\section{Participantes}

La población total del hospital durante la selección de la muestra en el año 2018 era de 1.384 individuos. De este conjunto se extrajo una muestra de 923 individuos ( $n=923$ ) que cumplía con los criterios de inclusión del estudio, seleccionando aquellos trabajadores sanitarios asistenciales que se encontrasen durante el año 2018 como parte activa de la plantilla y que el Servicio de Prevención (SP) tuviera información reciente sobre su estado serológico y vacunación, todo esto de forma independiente a su categoría profesional, su servicio de adscripción, edad y sexo. El personal que no contó con los criterios de inclusión fue excluido del estudio.

\section{Instrumentos}

Para poder recopilar la información se contó con los datos contenidos en el historial médico-laboral, así como los datos analíticos propios de los reconocimientos médico-laborales realizados por Salud Laboral mediante la realización de serologías, la revisión de las cartillas vacunales aportadas por los trabajadores, el registro de vacunas administradas por parte del servicio y la comprobación de los informes aportados por el resto de las especialidades médicas.

Para el análisis estadístico y de frecuencias de los datos se empleó la herramienta IBM SPSS en su versión 22, estos datos fueron previamente recogidos por el SP del Hospital de Poniente con carácter oficial, para la elaboración de los informes habituales emitidos como parte de la actividad propia del Servicio de Prevención.

\section{Procedimiento}

Antes de iniciar el artículo se presenta el diseño y el planteamiento del estudio a los miembros del Servicio de Prevención, siendo posteriormente presentado como proyecto al Comité de Ética e Investigación de la Provincia de Almería, donde fue aprobado.

Tras su aprobación, el equipo de investigación realiza una revisión de los casos de investigación en curso, manteniendo en todo momento la confidencialidad de los datos personales y de salud de los trabajadores. Para el manejo de la confidencialidad de la información, se identifica los casos mediante un número de identificación aleatorizado (ID), sin que se haga en ningún momento referencia expresa a los trabajadores objeto del estudio. Para aquellos casos de los que no se dispone de datos serológicos previos, se realizan las analíticas pertinentes durante los exámenes de salud planificados. Antes de iniciar dichos exámenes, los trabajadores firmaron su consentimiento mediante el cual acceden al uso de los datos necesarios para el desarrollo del proyecto.

Las variables necesarias y recopiladas por el Servicio de Prevención fueron tanto de tipo cuantitativo; edad (en años), como de tipo cualitativo; sexo (hombre/mujer), estado serológico frente al sarampión, la rubéola, la parotiditis, la varicela y la hepatitis $B$ (basándonos en niveles de inmunoglobulina $G(\lg G)$, pudiendo ser: positiva, dudosa o negativa), estado inmunitario del trabajador (distinguiendo entre: inmunidad natural, si está protegido tras el desarrollo de la enfermedad; inmunidad previa, si ya se le aplicó el calendario vacunal correctamente y si en la serología de los reconocimientos tiene anticuerpos positivos; o sin inmunidad, si lo anteriormente referido no ha sucedido y sus serologías son negativas o dudosas) o actuación por parte del SP (aplicación de la vacuna, recomendación de la vacuna o no se aplica pauta vacunal, en caso de que ya sea inmune).

Finalmente se analizó la seroconversión (IgG positivizada tras la vacunación) para aquellos casos en los que el SP administró pautas vacunales completas en aquellos trabajadores no inmunizados previamente y que se realizaron una serología posterior de confirmación.

\section{Análisis estadístico}

Todas las variables anteriormente referidas siguieron una distribución diferente a la normal (no paramétrica) por lo que en el análisis estadístico se aplicaron las pruebas de contraste pertinentes a dicha distribución, tomando como valor de significación estadística $p<0,05$. Para el análisis estadístico se usó principalmente el test de 
Kruskal Whallis con el fin de comprobar si los parámetros cuantitativos, como la edad, se encontraban relacionados con la posibilidad de que el sujeto presentase o no inmunidad.

\section{Resultados}

El estudió contó con una muestra de 923 casos $(n=$ $923)$, de los cuales el $74,2 \%(n=685)$ eran mujeres frente a un $25,8 \%$ de hombres $(n=238)$. La media de edad de la muestra fue de 41 años $(X=41,68)$ con una desviación estándar de 9,66 años (DE =9,66). Teniendo en cuenta la serología (Tabla 1) de los trabajadores, se pudo comprobar:

- Los niveles de lgG en rango protector para los microorganismos empleados en la Triple Vírica (TV) superan el $90 \%$ en todos los casos: 95,8 \% $(n=884)$ frente al sarampión, $91,3 \%$ para la rubéola $(n=843)$ y el $91,2 \%$ frente a la parotiditis $(n=842)$.

- Se presentaron los mayores niveles de protección del estudio para la varicela con un $96,5 \%(n=891)$ de los trabajadores sanitarios del estudio.

- El patógeno con menor nivel de protección presentado por los trabajadores fue la hepatitis $B$, con solo el $84,7 \%$ de la muestra con anticuerpos protectores en rango de inmunidad ( $n=782$ ) (Tabla 1$)$.

En todos los casos en los que se confirmó que el trabajador no presentaba inmunidad previa se recomendó la vacunación. Ésta se aplicó en aquellos casos en los que el trabajador consintió dicho procedimiento (Tabla 2):

- En el $1,9 \%$ del total de la muestra $(n=18)$ se pautó la TV para el sarampión, mientras que en el 2,1 $\%(n=19)$ del total de casos se vacunó debido a la falta de protección frente a la rubéola y el 3,5\% $(n=$ 32) necesitó su aplicación debido a la falta de respuesta inmunológica frente a la parotiditis. El total de vacunas de la TV administradas fue de 64, siendo 14 las que se administraron en el año 2018. La seroconversión del total de la muestra fue del $97,4 \%$ de los trabajadores no inmunizados frente al sarampión, 95,7 \% para la rubéola y $90,9 \%$ para aquellos casos no protegidos frente a parotiditis.

- El 2,4\% ( $n=22)$ del total de la población trabajadora sometida a este estudio se vacunó frente a la Varicela. En el último año, Salud Laboral indicó 5 vacunas antivaricela. La seroconversión de los casos del estudio fue del $77,3 \%$ de la muestra no inmunizada previamente frente a varicela.

- El mayor porcentaje de vacunados dentro de la población trabajadora del estudio fue para aquellos vacunados frente a la hepatitis $B$ con un $26 \%$ del total de los casos $(n=240)$. No se pautó vacunación frente a la hepatitis B durante el 2018. La seroconversión de los trabajadores fue del $82,1 \%$ de la muestra no inmunizada previa a la vacunación contra la hepatitis B (Tabla 2).

Tras la administración de las vacunas, un gran porcentaje de los trabajadores mostró unos títulos de anticuerpos en rango protector (Figura 1). En el caso de la Triple Vírica y la vacuna Anti-Varicela en todos los casos en los que no se desarrolló inmunidad, se aplicó una pauta incompleta de vacunación.

Tabla 1. Estado serológico actual de los trabajadores que componen la muestra del Hospital de Poniente.

\begin{tabular}{|l|c|c|c|c|c|}
\hline Serología (Ac) & Sarampión & Rubéola & Parotiditis & Varicela & Hepatitis B \\
\hline Positiva & $884(95,8 \%)$ & $843(91,4 \%)$ & $842(91,2 \%)$ & $891(96,5 \%)$ & $782(84,8 \%)$ \\
\hline Negativa & $25(2,6 \%)$ & $38(4,1 \%)$ & $57(6,2 \%)$ & $21(2,3 \%)$ & $137(14,8 \%)$ \\
\hline Dudosa & $14(1,6 \%)$ & $42(4,5 \%)$ & $24(2,6 \%)$ & $11(1,2 \%)$ & $4(0,4 \%)$ \\
\hline
\end{tabular}

Tabla de elaboración propia, con los datos serológicos de los trabajadores sanitarios del centro Hospital de Poniente obtenidos durante el desarrollo de los exámenes de salud realizados por el SP. 
Tabla 2. Cobertura vacunal de la muestra realizada por el Servicio de Prevención del Hospital de Poniente con los lotes más habituales.

\begin{tabular}{|c|c|c|c|c|}
\hline \multirow{2}{*}{ Vacuna } & \multicolumn{2}{|c|}{ No se administra vacunación } & Se administra vacunación \\
\cline { 2 - 5 } & $\begin{array}{l}\text { Inmunidad } \\
\text { previa }\end{array}$ & $\begin{array}{l}\text { Inmunidad } \\
\text { previa }\end{array}$ & $\begin{array}{l}\text { Sin } \\
\text { inmunidad } \\
\text { previa }\end{array}$ & \\
\cline { 2 - 5 } & Natural & Vacunal & 39 & 63 \\
\hline $\begin{array}{c}\text { Triple Vírica } \\
\text { (MMR VaxPro } 囚)\end{array}$ & 131 & 690 & 31 & 22 \\
\hline $\begin{array}{c}\text { Anti-Varicela } \\
\text { (Varivax®) }\end{array}$ & 254 & 616 & 141 & 240 \\
\hline $\begin{array}{c}\text { Anti-Hepatitis B } \\
\text { (HB VaxPro®) }\end{array}$ & 3 & 539 & & \\
\hline
\end{tabular}

Tabla de elaboración propia, con los datos sobre actuaciones en materia de vacunaciones administradas por el SP a los trabajadores sanitarios del centro Hospital de Poniente tras sus exámenes de salud.

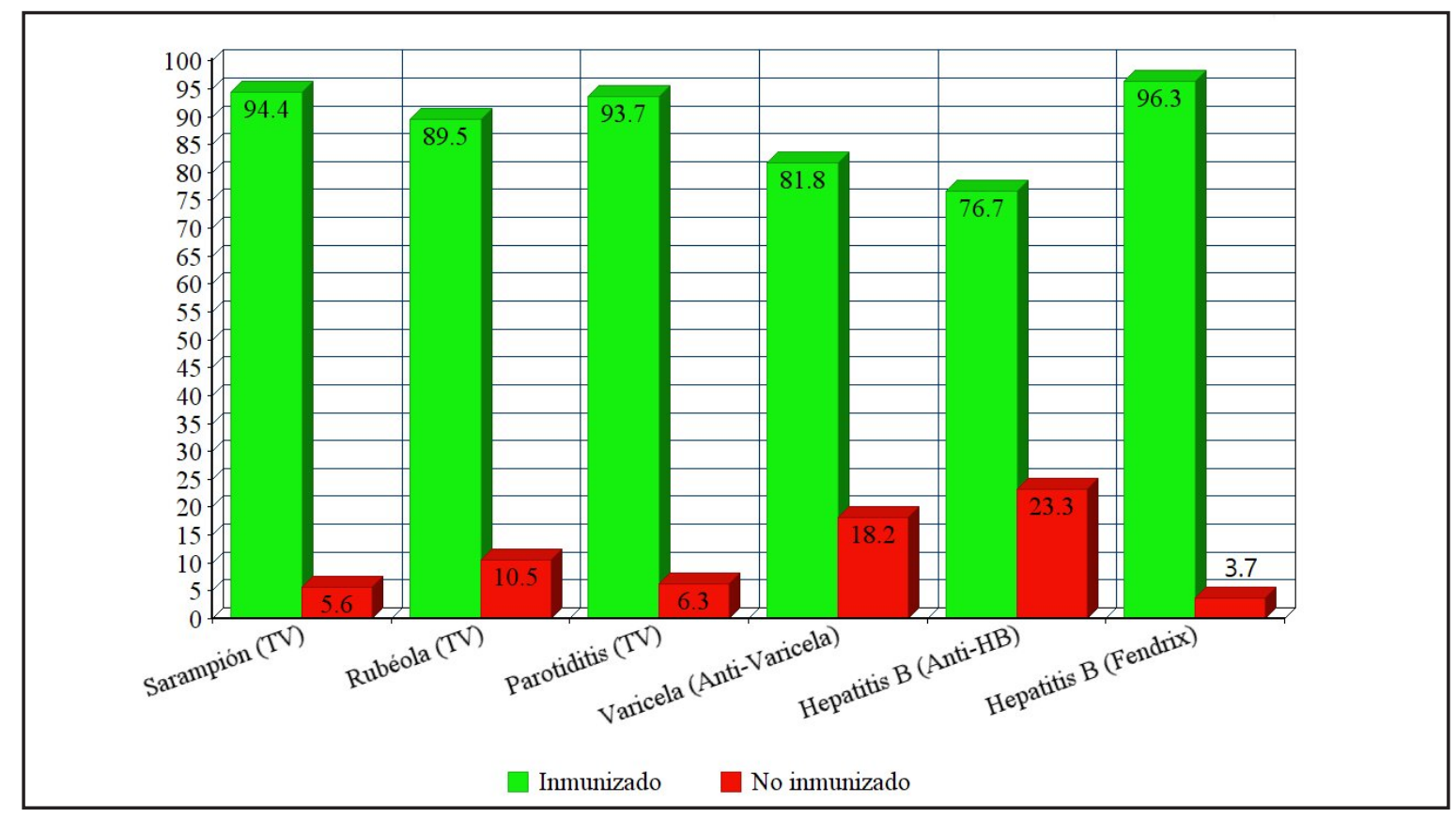

Figura 1. Cobertura vacunal realizada por el SP del Hospital de Poniente y porcentaje de respuesta inmunitaria de los trabajadores vacunados. 


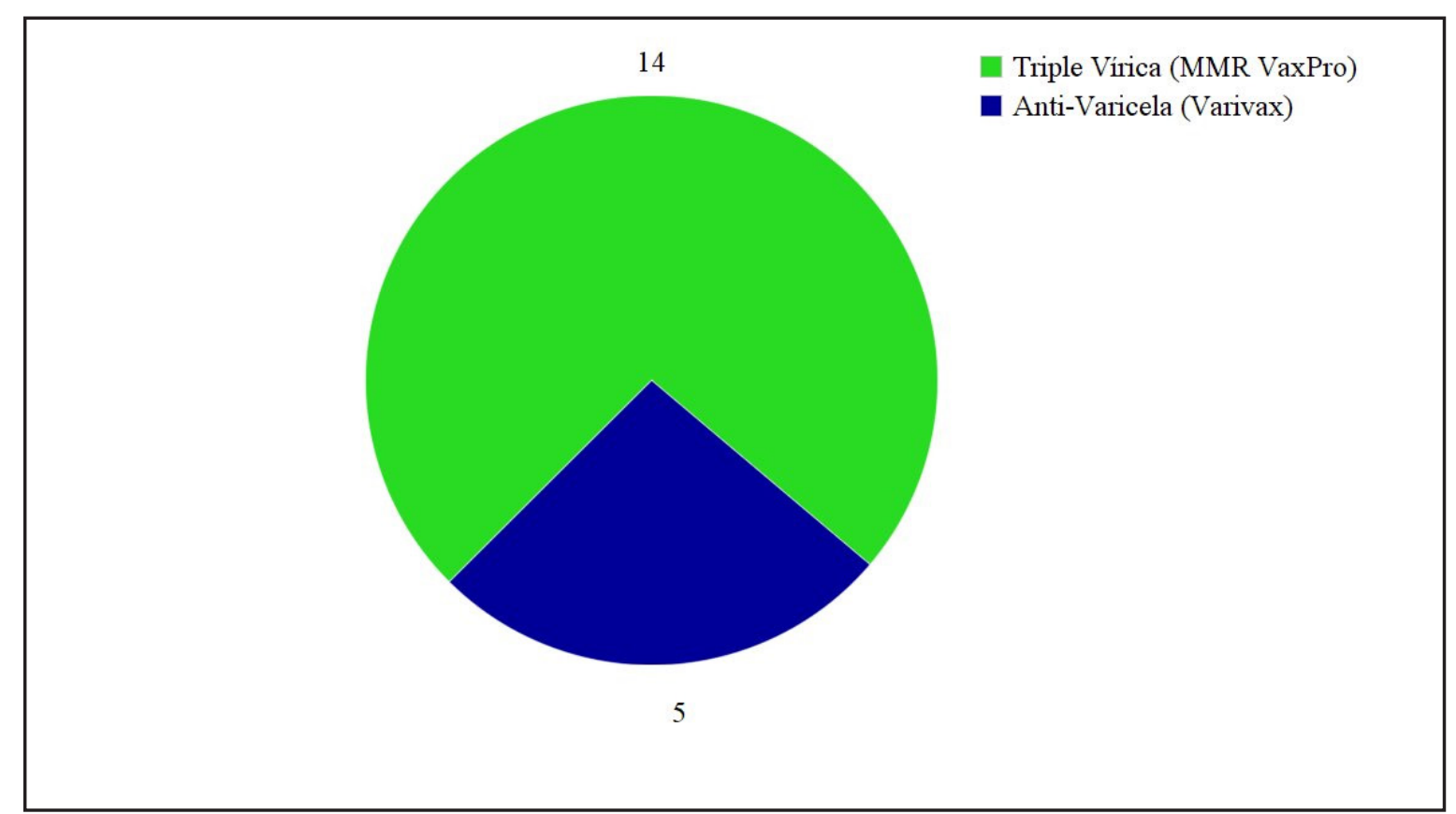

Figura 2. Vacunas administradas por el SP durante el año 2018.

\section{Análisis estadístico}

Se pudo observar diferencias estadísticamente significativas $(p<0,05)$ entre la serología, el estado inmunitario de la muestra y su edad para el caso del sarampión, la rubéola y la hepatitis $B$.

En el caso del sarampión y la rubéola, tras la aplicación del test de Kruskal Whallis, los grupos de mayor edad mostraron una serología protectora. Para ambos la media de edad fue aproximadamente de 42 años ( $X$ $=41,88$ años; $D E=9,63$ y $X=42,31$ años; $D E=8,45$ para sarampión y rubéola respectivamente). La serología negativa (no protectora) para ambos casos agrupaba en gran parte a un subgrupo más joven que el primero $(X=$ 36,96 años; $D E=9,16$ y $X=40,58$ años; $D E=8,49$ para sarampión y rubéola).

Atendiendo al otro patógeno referido anteriormente sucede lo contrario, tras emplear nuevamente el test de Kruskal Whallis, son un subgrupo de trabajadores de mayor edad los que se catalogaron como no inmunes frente a la hepatitis $B(X=45,71$ años; $D E=9,64)$, en contraposición con un subgrupo de trabajadores de menor edad, los cuáles sí que estaban correctamente protegidos frente al patógeno ( $X=40,98$ años; $D E=9,49)$.

\section{Discusión}

Inicialmente podríamos establecer que la cobertura vacunal y la protección serológica que presentan los trabajadores sanitarios de nuestro centro alcanza los objetivos que la comunidad sanitaria se fija para la población (Limia Sánchez \& Molina Olivas, 2015). Esto es posible debido a que muchos de nuestros trabajadores se encontraban previamente inmunes debido al alto porcentaje de los mismos que recibió dichas vacunas durante su infancia.

En aquellos trabajadores que no se encontraban inmunizados previamente, se constató un desarrollo de protección tras la aplicación de pautas vacunales completas por parte de nuestro SP. Hubo un alto porcentaje de seroconversión para todos los microorganismos, siendo menor en el caso de la parotiditis. Dichos resultados señalan a las vacunas como la principal medida preventiva frente al desarrollo de estas enfermedades y por tanto debe promoverse su uso.

Analizando al conjunto de los trabajadores, se observó un alto porcentaje de casos con una media de edad en torno a los 40 años, que presentaban inmunidad frente a los principales patógenos tales como el sarampión, la rubéola, la parotiditis o la varicela. Los resultados en los tres primeros patógenos pueden justificarse en gran medida gracias a la aplicación de la vacuna correspondiente, ya que cuando los trabajadores de dicha generación se encontraban en edad escolar, éstas ya eran de uso universal (Beleni \& Borgmann, 2018). Destacamos que al emplearse la misma vacuna contra estos tres primeros patógenos, podemos esperar que nuestros casos presenten unos estados serológicos 
similares entre ellos. No obstante, sí hemos podido identificar diferencias en la inmunidad de nuestros trabajadores sanitarios dependiendo de su grupo generacional, pudiendo encontrar una menor inmunidad frente al sarampión y a la rubéola en aquellos sanitarios más jóvenes en comparación con los de mayor edad. Esto podría deberse a varios motivos, como el inicio de los "Movimientos anti-vacunas" y su influencia durante la infancia de dicha generación (Phadke, Bednarczyk, Salmon \& Omer, 2016), así como el uso en el pasado de lotes de vacunas que presentaron una menor inmunogenicidad. Por otro lado, en el caso de la varicela, que cuenta con el mayor porcentaje de trabajadores inmunizados, puede deberse a una inmunidad tanto natural como vacunal (Peña-Rey et al., 2009) debido a que la vacuna se implantó tardíamente en nuestro calendario vacunal infantil.

Si bien, como ya hemos señalado, consideramos que se puede tratar de un fenómeno multi-etiológico (desde una menor vacunación en la infancia producto de la desinformación generalizada sobre los beneficios vacunales, hasta las propias características del individuo, que puede responder en menor medida a la vacuna por sus características individuales) por lo que debemos recordar que se debería poder actuar sobre aquellas causas que sí dependan íntegramente de nosotros. Para ello, resultados como los arrojados por este estudio deberían tener una mayor divulgación en la sociedad, con la finalidad de combatir la desinformación que algunos de los "Movimientos anti-vacunas" ejerce sobre la sociedad (Boyero García, 2016).

Al analizar el estado inmunológico de los trabajadores frente a la hepatitis $B$, observamos que el efecto es el inverso, siendo en aquellos trabajadores de menor edad en los que se presenta una serología protectora frente al agente infeccioso. Estos resultados coinciden con otros estudios previos, que señalan que al ser una enfermedad de menor prevalencia en la población general, con unos mecanismos de contagio que implican exposición a fluidos corporales contaminados, gran parte de la población no ha podido desarrollar inmunidad por exposición directa a la enfermedad. Gran parte de la inmunidad de nuestros casos se corresponde con un correcto proceso de vacunación, vacunas que en muchos otros países se empezaron a llevar a cabo en la población más joven. Aún así, dicha serología de inmunidad sigue siendo elevada, aunque notablemente inferior a las anteriores (Ni et al., 2012).

Al ser los trabajadores sanitarios un grupo de riesgo para todas estas patologías, el objetivo del SP del centro debe ser el de garantizar el mejor estado inmunitario de los trabajadores, por lo que se debe mantener una alta cobertura vacunal en aquellos que lo necesiten, así como promover la vacunación como principal medida preventiva.

La principal limitación del estudio ha sido establecer el origen de la protección inmunológica en todas las serologías, ya que en casos aislados no tuvimos acceso a fuentes objetivas como la cartilla de vacunación infantil o el historial médico completo del trabajador (pudiendo ser el origen natural de su inmunidad). Otros factores como el desabastecimiento de determinadas vacunas en momentos puntuales del estudio o la no asistencia de los trabajadores a sus exámenes de salud pudieron dificultar el acceso a la muestra.

\section{Conclusiones}

Este estudio pone de manifiesto la importancia de las vacunas como principal medida preventiva frente al desarrollo de enfermedades altamente contagiosas que están volviendo a resurgir en la población general, debido a múltiples factores entre los que se destaca un menor uso de las mismas. Como ya hemos referido previamente, el objetivo de todo SP debe ser el de ofrecer la máxima protección posible, logrando en nuestro caso, alcanzar más del $90 \%$ de serologías en rango de inmunoprotección para la gran mayoría de patógenos estudiados (siendo aquellos valores entre $90 \mathrm{y}$ $95 \%$ los señalados por los expertos como objetivo), ya sea por una correcta vacunación durante la infancia, como por la administración de la vacuna en aquellos adultos donde no se ha desarrollado inmunidad previa.

Aún así no debemos descuidar nuestra vigilancia sobre la salud de nuestros trabajadores, siendo de gran importancia, continuar con una vigilancia activa por parte de Salud Laboral, mediante la realización de serologías en el personal asistencial de nueva incorporación, así como, proceder a la vacunación en los casos necesarios y reforzar el mensaje mediante campañas informativas sobre las ventajas que presentan las vacunas. Todo esto refuerza nuestra visión de que la administración de vacunas supone a la larga un ahorro económico en costes directos e indirectos para el sistema de salud público. Es por ello que se deben seguir aplicando medidas como una mayor difusión de las ventajas de las mismas (con una mayor campaña informativa y divulgativa) a fin de impedir que movimientos como los anti-vacunas cobren fuerza en nuestra sociedad. 


\section{Agradecimientos}

A la totalidad del Servicio de Prevención de la Agencia Pública Sanitaria Poniente por su contribución con la revisión del estudio. También agradecemos enormemente a la Unidad de Apoyo a la Investigación de la Agencia Pública Sanitaria Poniente por su constante disposición a colaborar con los autores durante el desarrollo del estudio.

\section{Referencias Bibliográficas}

Amela, C. (2004). Epidemiología de las enfermedades incluidas en un programa de vacunación. Madrid, España: EMISA.

Arrazola Martínez, M., de Juanes Pardo, J., \& García de Codes Ilario, A. (2015). Conceptos generales. Calendarios de vacunación sistemática del niño y del adulto en España. Impacto de los programas de vacunación. Enfermedades Infecciosas $y$ Microbiología Clínica, 33(1), 58-65. doi: 10.1016/j. eimc.2014.12.001

Beleni, A., \& Borgmann, S. (2018). Mumps in the Vaccination Age: Global Epidemiology and the Situation in Germany. International Journal Of Environmental Research And Public Health, 15(8), 1618. doi: 10.3390/ijerph15081618

Botelho-Nevers, E., Cassir, N., Minodier, P., Laporte, R., Gautret, P., Badiaga, S, ... Brouqui, P. (2011). Measles among healthcare workers: a potential for nosocomial outbreaks. Eurosurveillance: Bulletin Europeen Sur Les Maladies Transmissibles, 16(2), 7-11.

Boyero García, R. (2016). ¿Vaccine hesitancy?: indecisión ante la vacunación. Factores determinantes y estrategias de intervención. Girona, España: Universitat de Girona.

Cohen, C., White, J.M., Savage, E.J., Glynn, J.R., Choi, Y., Andrews, N., ... Ramsay, M. E. (2007). Vaccine Effectiveness Estimates, 2004-2005 Mumps Outbreak, England. Emerging Infectious Diseases, 13(1), 12-7. doi: 10.3201/eid1301.060649

Kew, M. (2010). Epidemiology of chronic hepatitis $B$ virus infection, hepatocellular carcinoma, and hepatitis B virus-induced hepatocellular carcinoma. Pathologie Biologie, 58(4), 273-277. doi: 10.1016/j. patbio.2010.01.005

Limia Sánchez, A., \& Molina Olivas, M. (2015). Programa y coberturas de vacunación frente a sarampión y rubeola en España: retos para alcanzar su eliminación.
Revista Española de Salud Pública, 89(4), 357364. doi: 10.4321/s1135-57272015000400004

Ministerio de Sanidad, Consumo y Bienestar Social. (2019). Calendario acelerado de vacunaciones. Madrid: Comisión de Salud Pública del Consejo Interterritorial del SNS.

Ministerio de Sanidad, Consumo y Bienestar Social. (2018). Vacunación en población adulta. Madrid: Comisión de Salud Pública del Consejo Interterritorial del SNS.

Ministerio de Sanidad, Consumo y Bienestar Social. (2018). Vacunación en grupos de riesgo de todas las edades y en determinadas situaciones. Madrid: Consejo Interterritorial del Sistema Nacional de Salud.

Ministerio de Sanidad, Servicios Sociales e Igualdad. (2017). Vacunación en trabajadores sanitarios. Madrid: Comisión de Salud Pública del Consejo Interterritorial del SNS.

Ni, Y., Chang, M., Wu, J., Hsu, H., Chen, H., \& Chen, D. (2012). Minimization of hepatitis B infection by a 25-year universal vaccination program. Journal Of Hepatology, 57(4), 730-735. doi: 10.1016/j. jhep.2012.05.021

Peña-Rey, I., Martínez de Aragón, M., Villaverde Hueso, A., Terres Arellano, M., Alcalde Cabero, E., \& Suárez Rodríguez, B. (2009). Epidemiología de la varicela en España en los períodos pre y post vacunación. Revista Española de Salud Pública, 83(5), 711724. doi: 10.1590/s1135-57272009000500012

Phadke, V., Bednarczyk, R., Salmon, D., \& Omer, S. (2016). Association Between Vaccine Refusal and Vaccine-Preventable Diseases in the United States. JAMA, 315(11), 1149. doi: 10.1001/jama.2016.1353

Rosell Aguilar, I. (2017). "Antivacunas" y dudas parentales en vacunación infantil: recuperemos la confianza en los profesionales sanitarios. Clínica, 26, 3-10. doi: 10.24197/cl.26.2017.3-10

Salleras, L. (2018). Movimientos antivacunas: una llamada a la acción. Vacunas, 19(1), 1-3. doi: 10.1016/j.vacun.2018.03.001

Servicio de Prevención de la Agencia Pública Sanitaria Hospital de Poniente. (2013). Procedimiento de vacunación frente a hepatitis $B$ en los profesionales de la Agencia Sanitaria Poniente. El Ejido. 
52 // Tejada-Pérez et al.

Shepard, C. (2006). Hepatitis B virus infection: epidemiology and vaccination. Epidemiologic Reviews, 28(1), 112-125. doi: 10.1093/epirev/ mxj009

Torres González, O. (2018). Claves para comprender la resistencia de los colectivos antivacunas: una controversia científico-tecnológica pública. Revista de Humanidades de Valparaíso, (11)7, 7-37. doi: 10.22370/rhv.2018.11.1194

Zanetti, A., Van Damme, P., \& Shouval, D. (2008). The global impact of vaccination against hepatitis $B$ : A historical overview. Vaccine, 26(49), 6266-6273. doi: 10.1016/j.vaccine.2008.09.056 